\title{
Portlandite crystal: Bulk, bilayer, and monolayer structures
}

\author{
Y. Aierken, ${ }^{1}$ H. Sahin, ${ }^{1, *}$ F. Iyikanat, ${ }^{2}$ S. Horzum, ${ }^{1}$ A. Suslu, ${ }^{3}$ B. Chen, ${ }^{3}$ R. T. Senger,${ }^{2}$ S. Tongay, ${ }^{3}$ and F. M. Peeters ${ }^{1}$ \\ ${ }^{1}$ Department of Physics, University of Antwerp, 2610, Antwerp, Belgium \\ ${ }^{2}$ Department of Physics, Izmir Institute of Technology, 35430 Izmir, Turkey \\ ${ }^{3}$ School for Engineering of Matter, Transport and Energy Arizona State University, Tempe Arizona, 85287, USA
}

(Received 23 October 2014; revised manuscript received 1 May 2015; published 12 June 2015)

\begin{abstract}
$\mathrm{Ca}(\mathrm{OH})_{2}$ crystals, well known as portlandite, are grown in layered form, and we found that they can be exfoliated on different substrates. We performed first principles calculations to investigate the structural, electronic, vibrational, and mechanical properties of bulk, bilayer, and monolayer structures of this material. Different from other lamellar structures such as graphite and transition-metal dichalcogenides, intralayer bonding in $\mathrm{Ca}(\mathrm{OH})_{2}$ is mainly ionic, while the interlayer interaction remains a weak dispersion-type force. Unlike well-known transition-metal dichalcogenides that exhibit an indirect-to-direct band gap crossover when going from bulk to a single layer, $\mathrm{Ca}(\mathrm{OH})_{2}$ is a direct band gap semiconductor independent of the number layers. The in-plane Young's modulus and the in-plane shear modulus of monolayer $\mathrm{Ca}(\mathrm{OH})_{2}$ are predicted to be quite low while the in-plane Poisson ratio is larger in comparison to those in the monolayer of ionic crystal BN. We measured the Raman spectrum of bulk $\mathrm{Ca}(\mathrm{OH})_{2}$ and identified the high-frequency $\mathrm{OH}$ stretching mode $A_{1 g}$ at $3620 \mathrm{~cm}^{-1}$. In this study, bilayer and monolayer portlandite $\left[\mathrm{Ca}(\mathrm{OH})_{2}\right]$ are predicted to be stable and their characteristics are analyzed in detail. Our results can guide further research on ultrathin hydroxites.
\end{abstract}

DOI: 10.1103/PhysRevB.91.245413

PACS number(s): 81.16.Pr, 68.65.Pq, 71.15.Nc, 31.15.A-

\section{INTRODUCTION}

The synthesis of monolayer graphene [1-3] not only gave researchers, for the first time, access to a quasi-twodimensional (2D) crystal, but it also drew significant attention to similar single-atomic-layer structures such as silicene [4,5], germanene [5-7], and transition-metal dichalcogenides (TMDCs) [8,9]. Following advances in delamination, techniques of bulk materials opened the possibility of the synthesis of novel monolayer structures having new functionalities and their use in various fields of nanotechnology. In contrast to the abundant literature on graphene-like ultrathin structures, few-layer alkaline-earth-metal hydroxides (AEMHs) have not been investigated so far. Bulk forms of AEMHs are layered structures belonging to the $P \overline{3} m 1$ space group [10] and the crystal structure of a layered AEMHs comprises stacked sheets of $\mathrm{MO}_{6}(M=$ alkaline-earth metals $)$ edge-sharing octahedra (see Fig. 1). At each corner of an octahedron, each $\mathrm{O}$ atom binds one $\mathrm{H}$ atom and the latter interacts with three neighboring hydroxyl groups of the adjacent layer. Early studies on bulk AEMHs revealed that the application of temperature and pressure may result in dramatical changes in their crystal structure and their electronic properties [11-18]. Moreover, early theoretical studies showed the reliability of the use of first principles calculations with a plane-wave basis set in combination with the generalized gradient approximation exchange-correlation functional for the investigation of structural and electronic properties of these materials [19-22].

Although the structural and electronic properties of bulk AEMHs have been investigated before [21,23,24], single layers of these materials have never been studied before and the their stability is still an open question. However, advances in experimental techniques made exfoliation and growth of such structures possible [25,26]. In particular,

*hasan.sahin@uantwerpen.be although the portlandite material has been the main product of hydration of portland cement and is one of the mostwell-known AEMHs, the characteristic properties of ultrathin structures of portlandite have not been reported yet. In this study we, experimentally and theoretically investigate the structural, electronic, magnetic, vibrational, and mechanical characteristics of bulk, bilayer, and monolayer $\mathrm{Ca}(\mathrm{OH})_{2}$ and discuss possible future applications.

This paper is organized as follows: In Sec. II we give details of our computational methodology. Our comparison of different systems on the structural, electronic, and mechanical properties are presented in Secs. III, IV, and V, respectively. Phonon dispersion and vibrational properties of monolayer structure are presented in Sec. VI. Our results are discussed in Sec. VII.

\section{COMPUTATIONAL METHODOLOGY}

Our first-principles calculations were carried out in the framework of density functional theory as implemented in the Vienna ab initio simulation package (VASP) [27-30]. For the exchange-correlation potential, the generalized gradient approximation (GGA) within the Perdew-Burke-Ernzerhof (PBE) formalism was employed [31,32]. The projector augmented wave (PAW) method [33,34] and a plane-wave basis set were used in the calculations.

A $\Gamma$-centered $k$-point mesh scheme was adapted for the Brillouin zone sampling. The $k$ points used in the density of states $($ DOS) calculations was $35 \times 35 \times 1$ for the monolayer and bilayer, and was $25 \times 25 \times 11$ for the bulk. The cutoff energy for the plane-wave basis was set to $500 \mathrm{eV}$. A Gaussian smearing method was employed and the width of the smearing was chosen to be $0.1 \mathrm{eV}$ in the DOS calculations and $0.01 \mathrm{eV}$ in the band-structure calculations. The convergence criterion of the self-consistent field calculations was set to $10^{-5} \mathrm{eV}$ for the total energy. A large vacuum spacing (at least $25 \AA$ ) was introduced in the unit cell to prevent the interlayer interaction 
(a)

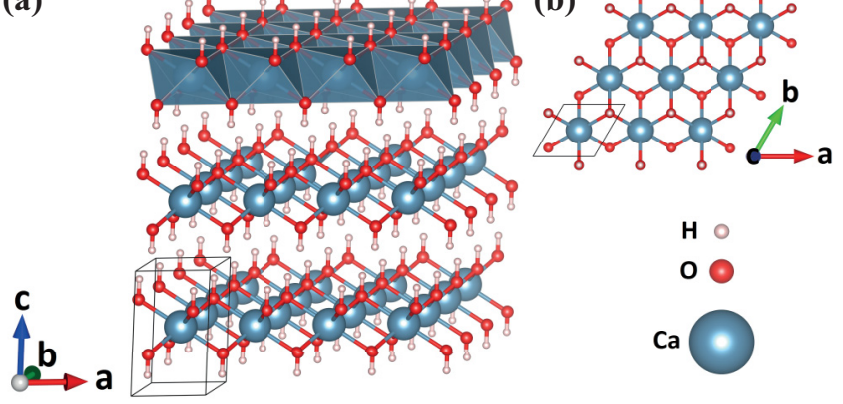

FIG. 1. (Color online) Atomic structure of bulk $\mathrm{Ca}(\mathrm{OH})_{2}$ : (a) tilted view, (b) top view of one layer.

in monolayer and bilayer calculations. The conjugate gradient method determined how the positions of the ions were updated until the atomic forces were less than $0.01 \mathrm{eV} / \AA$. Pressure on the supercells was decreased to a value less than $1 \mathrm{kB}$. A van der Waals (vdW) correction to the GGA functional was included by using the DFT-D2 method of Grimme [35] as implemented in VASP. Our charge analysis was performed by using a program written by Henkelman et al. which implemented Bader's charge analysis method [36-38].

Elastic constants are calculated in the linear regime of the stress-strain relationship by using a $1 \times 1$ unit cell: $\sigma=\boldsymbol{C \epsilon}$, where $\sigma$ is true stress (Cauchy stress), which is one of the outputs from VASP. Throughout this paper, all stresses in all structures are redefined as force per unit length by multiplying the true stress (force per unit area) by their $\mathbf{c}$ lattice constants perpendicular to the layer plane; this will give us comparable quantities, which reflect the mechanical properties of each layer in various structures. $\boldsymbol{\epsilon}$ is the engineering strain (Cauchy strain), which is the ratio of the total deformation to the initial dimensions of the material. For the linear relation to be valid, every component of $\epsilon$ is set to be less than 0.01 , where we confirmed the linearity. Applying the symmetry constrains for a hexagonal crystal structure, we obtain the following linear equations that have to be solved in order to calculate the stiffness matrix $\mathbf{C}$, where the Voigt notations are applied $(1 \rightarrow \mathrm{xx}, 2 \rightarrow \mathrm{yy}, 3 \rightarrow \mathrm{zz}, 4 \rightarrow \mathrm{yz}, 5 \rightarrow \mathrm{zx}, 6 \rightarrow \mathrm{xy})$ [39]:

$$
\left(\begin{array}{l}
\sigma_{1} \\
\sigma_{2} \\
\sigma_{6}
\end{array}\right)=\left(\begin{array}{ccc}
C_{11} & C_{12} & 0 \\
C_{12} & C_{11} & 0 \\
0 & 0 & \left(C_{11}-C_{12}\right) / 2
\end{array}\right)\left(\begin{array}{l}
\epsilon_{1} \\
\epsilon_{2} \\
\epsilon_{6}
\end{array}\right) .
$$

By least-squares fitting of the stress-strain data set from the application of particular uniaxial strain, it is possible to extract all coefficients. Using the Voigt notation, elastic constants can be calculated from the following relations [40]. Young's modulus, which is a measurement of the stiffness of the material and gives in particular the material's response to tensile or compressive stress, is given by

$$
E_{\alpha}=\frac{1}{S_{\alpha \alpha}}, \quad \alpha=1,2,3 .
$$

The Poisson ratio, which is the ratio of the transverse to the axial strain, represents how easy it is to change the shape with respect to the volume of the material. Liquid and rubber have a Poisson ratio close to 0.5 , which is the theoretical upper limit of this quantity. We can calculate Poisson's ratio by using the formula

$$
v_{\alpha \beta}=-E_{\beta} S_{\alpha \beta}, \quad \alpha, \beta=1,2,3(\alpha \neq \beta) .
$$

Shear modulus, which is also related to the stiffness of the material, i.e., it gives the material's response to shear stress, can be obtained from

$$
G_{\gamma \gamma}=\frac{1}{S_{\gamma \gamma}}, \quad \gamma=4,5,6,
$$

where $\mathbf{S}=\mathbf{C}^{-1}$ is the compliance matrix.

Moreover, for the calculation of the in-plane stiffness of $\mathrm{Ca}(\mathrm{OH})_{2}$ we focused on the harmonic range of the elastic deformation, where the structure responded linearly to strain $\epsilon$. The stretching of the $\mathrm{Ca}(\mathrm{OH})_{2}$ is achieved by increasing the equilibrium lattice constant $\mathrm{c}_{0}$ by $\Delta \mathrm{c}$, to attain the axial strain $\epsilon=\Delta \mathrm{c} / \mathrm{c}_{0}$. We optimized the atomic structure at each increment of the strain, $\Delta \epsilon=0.01$, and calculated the total energy under strain $\mathrm{E}_{T}(\epsilon)$. Then the strain energy can be given by $\mathrm{E}_{S}=\mathrm{E}_{T}(\epsilon)-\mathrm{E}_{T}(\epsilon=0)$; namely, the total energy at a given strain $\epsilon$ minus the total energy at zero strain. Then, by using the following formula, one can calculate the in-plane stiffness:

$$
C=\left(\frac{1}{A_{0}}\right)\left(\frac{d^{2} E_{S}}{d \epsilon^{2}}\right),
$$

where $A_{0}$ is the equilibrium area of the supercell.

As explained in detail in the following sections, unit cells including one $\mathrm{Ca}$, two $\mathrm{O}$, and two $\mathrm{H}$ are the primitive cells of both monolayer and bulk structures; it is doubled for bilayer. Cohesive energy per unit cell $E_{\mathrm{coh}}$ is calculated according to the formula: $E_{\mathrm{coh}}=E_{\mathrm{tot}}-n E_{C a}-2 n E_{\mathrm{O}}-2 n E_{\mathrm{H}}$, where $E_{\text {tot }}$ is the total energy of the unit cell of $\mathrm{Ca}(\mathrm{OH})_{2}, E_{X}$ is the single-atom total energy of atom $X$ and $n$ is the number of $\mathrm{Ca}$ atoms for the corresponding unit cell, i.e., $n=1, n=2$, and $n=1$ for monolayer, bilayer, and bulk, respectively.

In addition, the calculation of the desorption energies were performed on a $4 \times 4$ supercell by considering the spin-polarized ground state of the single $\mathrm{H}$ atom and $\mathrm{H}$-vacant site on the surface.

\section{STRUCTURAL PROPERTIES OF $\mathrm{Ca}(\mathrm{OH})_{2}$}

Before our theoretical investigation of few-layer $\mathrm{Ca}(\mathrm{OH})_{2}$, first we present the experimental realization and detailed theoretical analysis of the characteristics of bulk $\mathrm{Ca}(\mathrm{OH})_{2}$ crystals.

$\mathrm{Ca}(\mathrm{OH})_{2}$ crystals were grown using the hydrolysis technique by using $\mathrm{Ca}_{3} \mathrm{SiO}_{5}$ micropallets. $\mathrm{Ca}_{3} \mathrm{SiO}_{5}$ was mixed at different water-to-solid ratios ranging from 0.2 to 0.9 by molar weight. The mixture was heated up to $40^{\circ} \mathrm{C}$ in a controlled reaction chamber for 3 hours and controllably cooled down to $5^{\circ} \mathrm{C}$ for 24 hours by using a temperature controller. The growth time depends on the total water-to-solid ratio as well as the growth temperature. Growth time was around 8 hours for 0.6 water-to-solid ratio and $40^{\circ} \mathrm{C}$ growth temperature. Longer growth time typically resulted in a dendritic morphology where the growth was mostly in the $c$-axis direction. Synthesized crystals displayed sharp XRD (001) reflections at 19.1, 34, 56.2, 77.7 degrees implying that crystals have lamellar nature. 

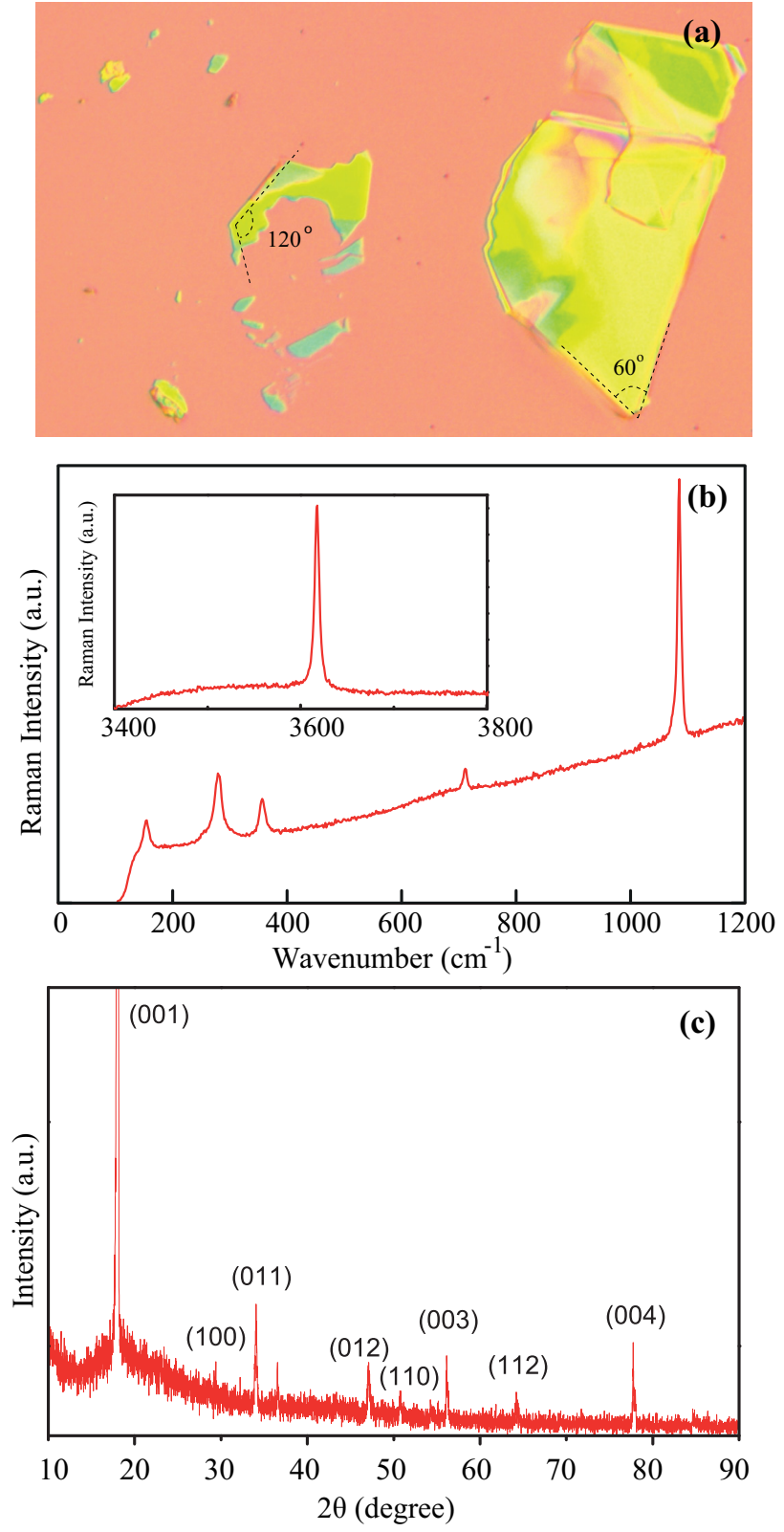

FIG. 2. (Color online) (a) Optical image of the crystal structure and (b) Raman spectrum measured using $488 \mathrm{~nm}$ laser in the low- and the high-frequency region. The fundamental phonon branches located at the low frequency (100-400 $\mathrm{cm}^{-1}$ range) and the high frequency $\left(3620 \mathrm{~cm}^{-1}\right)$ are associated with the $\mathrm{OH}$ stretching mode. (c) XRD measurements.

Synthesized crystals were around $0.1-2 \mathrm{~mm}$ in size and they were filtered from the solution. After the filtering process, crystallites were washed off by using $18.2 \mathrm{M} \Omega \mathrm{cm}$ DI wafer multiple times and dried under inert Ar gas. Crystallites were exfoliated by using a micromechanical exfoliation technique onto thermal oxide on silicon substrates. We find that the contrast was improved for an oxide thickness around 265$285 \mathrm{~nm}$. Exfoliated flakes displayed rather sharp edges (see Fig. 2) with well-defined angles of $120^{\circ}$ and $60^{\circ}$ implying that the materials are highly crystalline. Synthesized $\mathrm{Ca}(\mathrm{OH})_{2}$ flakes are in layered form and these flakes can be easily
TABLE I. Comparison of calculated results for structures parameter of bulk $\mathrm{Ca}(\mathrm{OH})_{2}$ with experimental results and with theoretical results from other references: lattice constants $a$ and $c$, volume $V$, and $c / a$ ratio.

\begin{tabular}{lcccc}
\hline \hline $\begin{array}{l}\text { Structure } \\
\text { parameters }\end{array}$ & Expt. $^{\mathrm{a}}$ & Expt. $^{\mathrm{b}}$ & $\begin{array}{l}\text { PBE-PAW } \\
\text { (this work) }\end{array}$ & PBE-PAW $^{\mathrm{c}}$ \\
\hline$a(\AA)$ & 3.589 & 3.592 & 3.614 & 3.612 \\
$c(\AA)$ & 4.911 & 4.906 & 4.982 & 4.942 \\
$V\left(\AA^{3}\right)$ & 54.78 & 54.82 & 56.35 & 55.85 \\
$c / a$ & 1.368 & 1.366 & 1.379 & 1.368 \\
\hline
\end{tabular}

a Ref. [41].

${ }^{\mathrm{b}}$ Ref. [42].

${ }^{\mathrm{c}}$ Ref. [23].

exfoliated by using the Scotch tape technique on different substrates. The exfoliated flakes do not show any signs of structural imperfection, pit formation, and overall rather flat surfaces can be obtained. In Fig. 2, the yellowish-looking regions actually correspond to regions where the thickness is around 50-100 $\mathrm{nm}(50-100$ layers) while the blue features are only $10-50 \mathrm{~nm}$ in thickness. Considering the ease to exfoliate this material, experimentally and theoretically we predict that they can be eventually isolated down to monolayers and few layers on various substrates.

In addition, micro-Raman measurements were performed using a $488 \mathrm{~nm}$ laser on a 2-micron-square spot by using a high-intensity laser of $10 \mathrm{~mW}$. We noticed that few-layers of $\mathrm{Ca}(\mathrm{OH})_{2}$ were not subject to local overheating or decomposition effects unlike TMDCs $\left(\mathrm{MoS}_{2}, \mathrm{WS}_{2}\right.$, etc.), which typically decompose around $100 \mu \mathrm{W}$ power using a similar laser excitation spot. We attribute this to the low absorption of the material associated with the rather large band gap. Raman measurements displayed several peaks in the $150-1000 \mathrm{~cm}^{-1}$ range. The high-frequency peak at $3620 \mathrm{~cm}^{-1}$ is associated with the $\mathrm{O}-\mathrm{H}$ stretching mode $A_{1 g}$.

Here, we note that, even though this material is a direct band gap semiconductor, its band gap is well beyond our detectors range and, since the insulators cannot be excited with such high laser wavelength, photoluminescence (PL) measurements are virtually impossible.

The bulk structure of portlandite is formed by the stacking of individual $\mathrm{Ca}(\mathrm{OH})_{2}$ monolayers on top of each other; see Fig. 1. As we exam and discuss the stacking in detail in the following paragraphs, we learned that the AA stacking is the ground-state atomic configuration for bulk and multilayer structures of $\mathrm{Ca}(\mathrm{OH})_{2}$. In Table I, optimized lattice parameters of the bulk structure together with experiments and other theoretical calculations are presented. Our results are consistent with Ref. [23] and together they have good agreement with experiments. This justifies the reliability of our calculations.

In the five-atomic hexagonal primitive unit cell of bulk $\mathrm{Ca}(\mathrm{OH})_{2}$, the $\mathrm{Ca}$ atom sits at the geometrical center of the cell, i.e., $\{1 / 2 a, 1 / 2 b, 1 / 2 c\}$. Two $\mathrm{O}$ and two $\mathrm{H}$ atoms form two hydroxyl groups $\left(\mathrm{OH}^{-}\right)$located symmetrically with respect to the $\mathrm{Ca}$ atom. In this arrangement, coordinates of $\mathrm{H}$ and $\mathrm{O}$ only differ by their positions along the $\mathbf{c}$ lattice axis and their fractional coordinates can be given as $\{1 / 6 a, 1 / 6 b,(1 / 2 c-$ $\left.\left.c_{\mathrm{O}}\right)\right\}$ and $\left\{1 / 6 a, 1 / 6 b,\left(1 / 2 c-c_{\mathrm{H}}\right)\right\}$ for one hydroxyl, 
TABLE II. Calculated results for different structures of $\mathrm{Ca}(\mathrm{OH})_{2}$ : lattice constants $a$, vertical shift of $\mathrm{O}$ and $\mathrm{H}$ atoms $\mathrm{c}_{\mathrm{O}}$ and $\mathrm{c}_{\mathrm{H}}$, $\mathrm{Ca}-\mathrm{O}$ and $\mathrm{O}-\mathrm{H}$ bond lengths, energy band gap $E_{\mathrm{gap}}$, cohesive energy per atom $E_{\mathrm{coh}}$, charge transfer $\Delta Q$ from Ca atom to $\mathrm{O}$ atom, in-plane Young's modulus $E_{x x}, E_{y y}$, in-plane Poisson ratio $v_{x y}$, in-plane shear modulus $G_{x y}$, and in-plane stiffness $C$. For comparison, theoretical calculations on same quantities of $\mathrm{BN}$ are shown in the last row.

\begin{tabular}{|c|c|c|c|c|c|c|c|c|c|c|}
\hline & $\begin{array}{c}a \\
(\AA)\end{array}$ & $\begin{array}{l}\mathrm{c}_{\mathrm{O}} / \mathrm{c}_{\mathrm{H}} \\
(\AA / \AA)\end{array}$ & $\begin{array}{c}\mathrm{Ca}-\mathrm{O} / \mathrm{O}-\mathrm{H} \\
(\AA / \AA)\end{array}$ & $\begin{array}{l}E_{\text {gap }} \\
(\mathrm{eV})\end{array}$ & $\begin{array}{l}E_{\text {coh }} \\
(\mathrm{eV})\end{array}$ & $\begin{array}{l}\Delta Q \\
(\mathrm{e})\end{array}$ & $\begin{array}{c}E_{x x}, E_{y y} \\
(\mathrm{~N} / \mathrm{m})\end{array}$ & $v_{x y}$ & $\begin{array}{c}G_{x y} \\
(\mathrm{~N} / \mathrm{m})\end{array}$ & $\begin{array}{c}C \\
\left(\mathrm{~J} / \mathrm{m}^{2}\right)\end{array}$ \\
\hline Bulk Ca $(\mathrm{OH})_{2}$ & 3.61 & $1.15 / 2.12$ & $2.36 / 0.97$ & 4.08 & 4.52 & 1.6 & 55.0 & 0.30 & 21.23 & 60.1 \\
\hline $2 \mathrm{~L} \mathrm{Ca}(\mathrm{OH})_{2}$ & 3.62 & $1.15 / 2.12$ & $2.38 / 0.97$ & 3.70 & 4.48 & 1.6 & 50.7 & 0.32 & 19.16 & 55.6 \\
\hline $1 \mathrm{~L} \mathrm{Ca}(\mathrm{OH})_{2}$ & 3.62 & $1.14 / 2.11$ & $2.38 / 0.97$ & 3.67 & 4.39 & 1.6 & 50.7 & 0.33 & 19.08 & 53.2 \\
\hline $1 \mathrm{~L} \mathrm{BN}$ & $2.51^{\mathrm{a}}$ & & $1.45^{\mathrm{a}, \mathrm{b}}$ & $4.64^{\mathrm{a}}$ & $8.82^{\mathrm{a}}$ & $0.43^{\mathrm{a}, \mathrm{c}}$ & $278.2^{\mathrm{d}}$ & $0.22^{\mathrm{d}}$ & $113.5^{\mathrm{d}}$ & $267^{\mathrm{e}}$ \\
\hline
\end{tabular}

${ }^{\mathrm{a}}$ Ref. [43].

${ }^{\mathrm{b}} \mathrm{B}-\mathrm{N}$ bond length.

${ }^{\mathrm{c}}$ Charge transfer from $\mathrm{B}$ to $\mathrm{N}$.

${ }^{\mathrm{d}}$ Ref. [44].

${ }^{\mathrm{e}}$ Ref. [7].

$\left\{5 / 6 a, 5 / 6 b,\left(1 / 2 c+c_{\mathrm{O}}\right)\right\}$ and $\left\{5 / 6 a, 5 / 6 b,\left(1 / 2 c+c_{\mathrm{H}}\right)\right\}$ for the other one, where $c_{O}$ and $c_{H}$ are the vertical shifts of the positions of $\mathrm{O}$ and $\mathrm{H}$ atoms from the $\mathrm{Ca}$ plane in $\AA$, respectively.

In the optimized structure, the lattice constants $a$ and $c$ are $3.61 \AA$ and $4.98 \AA$ in the bulk structure, parameters $c_{O}$ and $\mathrm{c}_{\mathrm{H}}$ are calculated to be 1.15 and $2.12 \AA$, see Table II. Bond lengths of $\mathrm{Ca}-\mathrm{O}$ and $\mathrm{O}-\mathrm{H}$ are 2.36 and $0.97 \AA$, respectively. Interlayer distance which is defined as the distance between the uppermost $\mathrm{H}$-layer of the underlying layer and the lowermost $\mathrm{H}$ layer of the top-lying layer is found to be $0.49 \AA$. Differing from other lamellar bulk crystal structures such as graphite $(3.58 \AA)$ and $\mathrm{MoS}_{2}(3.42 \AA)$ [45], $\mathrm{Ca}(\mathrm{OH})_{2}$ layers are more closely stacked on top of each other.

Our calculations reveal that going from bulk to monolayer, the in-plane lattice parameter $a$ change to $3.62 \AA$. In our calculations, the $c$ lattice parameter in the hexagonal unit cell of the monolayer is set to $25 \AA$ in order to avoid interlayer interaction between the adjacent layers. In the monolayer $\mathrm{Ca}(\mathrm{OH})_{2}$, parameters $\mathrm{c}_{\mathrm{O}}$ and $\mathrm{c}_{\mathrm{H}}$ are calculated to be $c_{\mathrm{O}}=1.14 \AA$ and $\mathrm{c}_{\mathrm{H}}=2.11 \AA$, respectively. $\mathrm{Ca}-\mathrm{O}$ and $\mathrm{O}-\mathrm{H}$ bond distances are 2.38 and $0.97 \AA$ in the monolayer, respectively. We observed only quite small change as the system goes from bulk to monolayer, some of the structure parameters even left unchanged, from this we can conclude quite weak interlayer interaction in $\mathrm{Ca}(\mathrm{OH})_{2}$. In order to study the interlayer interaction we further investigate their effect on stacking, and by including the vdW correction in the functional we are able to identify the nature of this interaction.

Individual layers of lamellar structures such as graphite, hex-BN, and TMDCs are held together mainly by the vdW force in order to form a bulk layered structure. Such a weak interaction stems from dynamical correlations between fluctuating charge distributions in neighboring layers. Here we investigated the energies of various bilayer configurations. As presented in Fig. 3 there are six possible types of stacking between two $\mathrm{Ca}(\mathrm{OH})_{2}$ monolayers. Similar to the stacking nomenclature of bilayer graphene, we classify the stacking types to be either $\mathrm{AA}$ or $\mathrm{ABn}(n=1,2, \ldots, 5)$. The same type of atoms from different monolayers are on top of each other in AA stacking whereas AB stackings can be reached by shifting one of the layers along certain lattice vectors. One set of $\mathrm{AB}$ stackings could be realized by shifting the second layer in the AA stacking towards [110], which gives stacking AB1, and by shifting towards the [110] direction, which gives stacking AB2; see first row in Fig. 3. Another set of bilayers are achieved by first flipping the second layer upside down in AA stacking, which would gives stacking $\mathrm{AB} 3$, then $\mathrm{AB} 4$ and $\mathrm{AB} 5$ can be constructed by doing the same shifting on the second layer of AB3 towards the [110] and [110] directions of the first layer, respectively. After
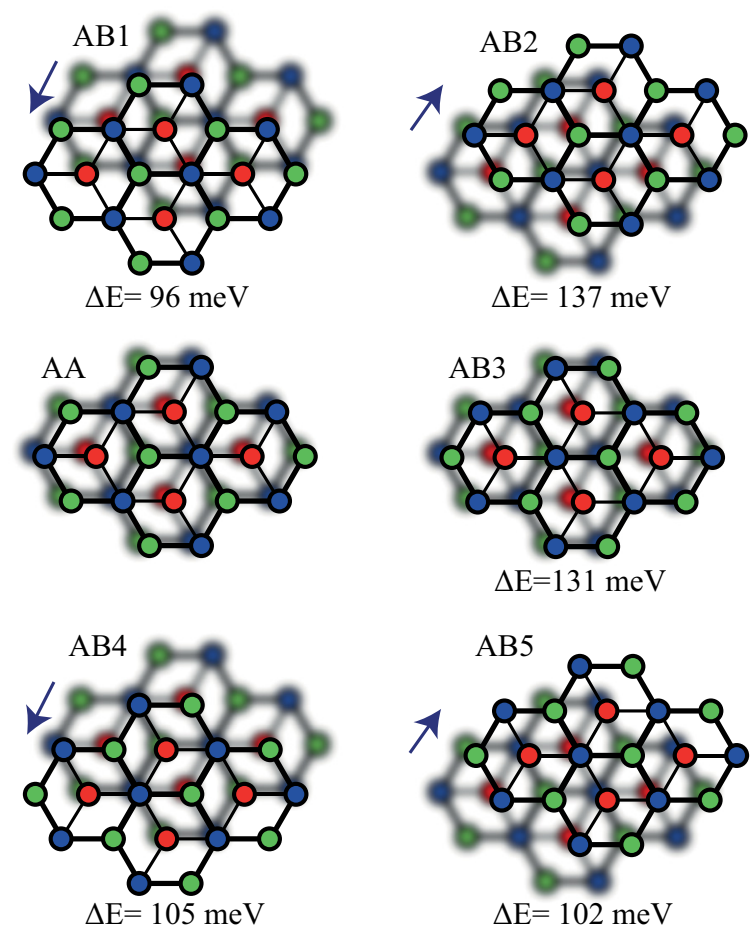

FIG. 3. (Color online) Different stacked bilayers (bottom layer is blurred) and their energy difference with respect to the AA stacking of $\mathrm{Ca}(\mathrm{OH})_{2}$, i.e., $\Delta E=E_{\mathrm{ABX}}-E_{\mathrm{AA}},(X=1,2, \ldots, 5)$. Energies are given per formula of $\mathrm{Ca}(\mathrm{OH})_{2}$. Blue, green, and red circles are stand for $\mathrm{Ca}$ atoms, the upper hydroxyl group, and the lower hydroxyl group, respectively. For clarity, the bottom layer is shifted slightly. 


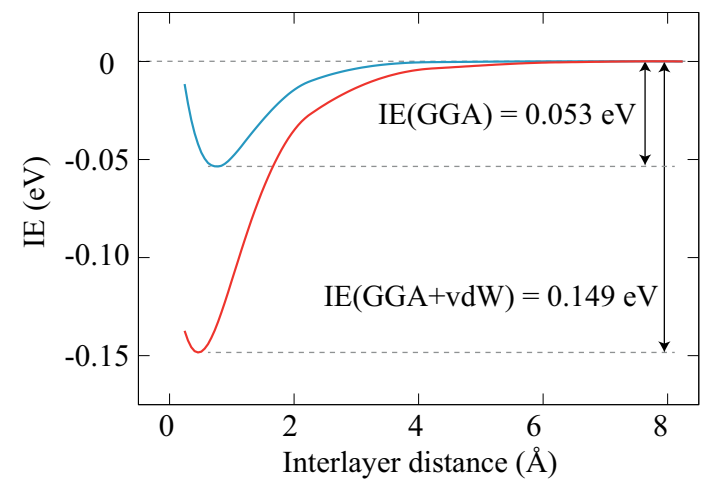

FIG. 4. (Color online) Interlayer interaction energy per formula of AA-stacked bilayer $\mathrm{Ca}(\mathrm{OH})_{2}$. Blue and red curves are for GGA calculations without and with vdW correction, respectively.

relaxation of all stackings, the variation of the a lattice constant among the different stacking types is less than $0.01 \AA$. The smallest interlayer distance, as defined previously for bulk, is for AA stacking which equals $0.49 \AA$, the same as that in bulk. For $\mathrm{AB}$ stacking the interlayer distance is $1 \sim 2 \AA$ larger than that for AA stacking. As depicted in Fig. 3, AA stacking is $96 \sim 137 \mathrm{meV}$ per formula more favorable than all other possible stacking types and hence it corresponds to the lowest-energy configuration.

To investigate the nature of the interlayer interaction, we calculated the interlayer interaction energy (IE) for the AA stacked bilayer structure of $\mathrm{Ca}(\mathrm{OH})_{2}$. The IE is the energy difference between the total energy at a specific interlayer distance and that of a well separated bilayer. The plot of IE versus interlayer distance is shown in Fig. 4, where the energy of the well-separated bilayer is defined as $0 \mathrm{eV}$. Two sets of calculations were performed, one set only considers GGA exchange correlation; while another set considers both the GGA and vdW interaction. At the optimized interlayer distance for the bilayer structure, almost two-thirds of the attractive interaction comes from the $\mathrm{vdW}$ interaction, and this is consist with D'Arco et al. (1993) [22]. They stated that the interlayer interaction of brucite, one of the isomorphous of portlandite, is mainly a dispersion-type interaction. The nature of interlayer interaction in $\mathrm{Ca}(\mathrm{OH})_{2}$ is mainly a vdW-type weak interaction. Our GGA + vdW calculations revealed that the interlayer interaction between two layers of $\mathrm{Ca}(\mathrm{OH})_{2}(149$ $\mathrm{meV}$ per formula) is much stronger than that of bilayers of $\mathrm{MoS}_{2}$ (76 meV per formula).

\section{ELECTRONIC PROPERTIES OF $\mathrm{Ca}(\mathrm{OH})_{2}$}

Our Bader charge transfer analysis showed that the final (initial) electron charge on $\mathrm{Ca}, \mathrm{O}$ and $\mathrm{H}$ atoms after (before) the formation of the crystal are $6.4 e(8.0 e), 7.4 e(6.0 e)$, and $0.4 e(1.0 e)$, respectively. Therefore, in the bulk structure of $\mathrm{Ca}(\mathrm{OH})_{2}, \mathrm{Ca}-\mathrm{O}$ bonds, which are mostly of ionic character, are formed through $0.8 e$ charge transfer from each $\mathrm{Ca}$ to $\mathrm{O}$ atom. Charge transfer is kept unchanged when it comes to the monolayer structure, except for the rest charge on $\mathrm{H}$ atom is $0.6 e$ in the monolayer.

Our calculations on the electronic structure reveal that bulk $\mathrm{Ca}(\mathrm{OH})_{2}$ is an insulator with a $4.37 \mathrm{eV}$ direct band gap. (a)

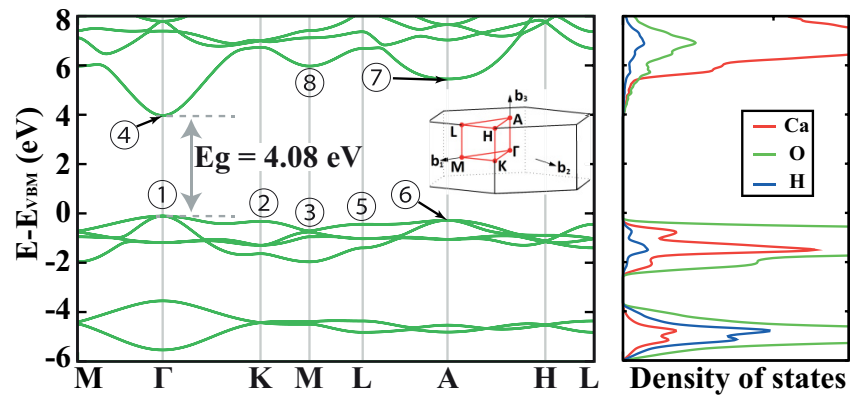

(c)
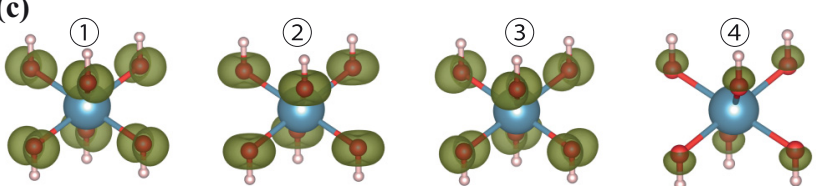

(5)
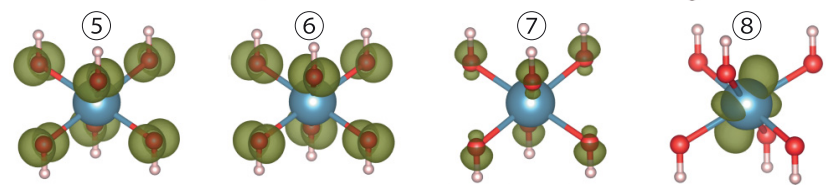

(d)

(e)

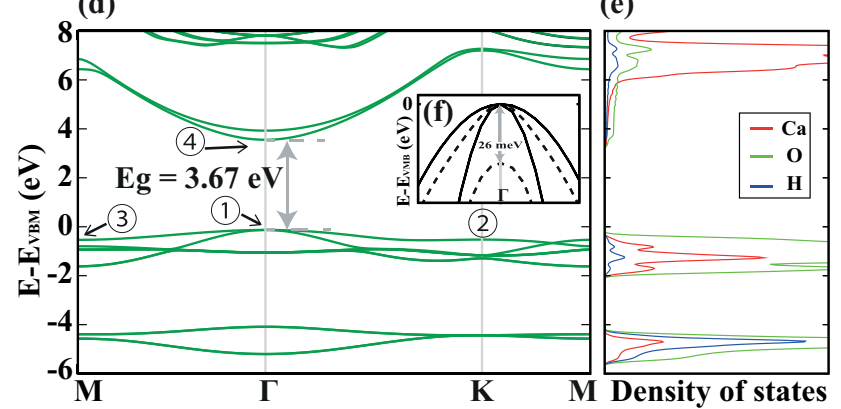

(g)
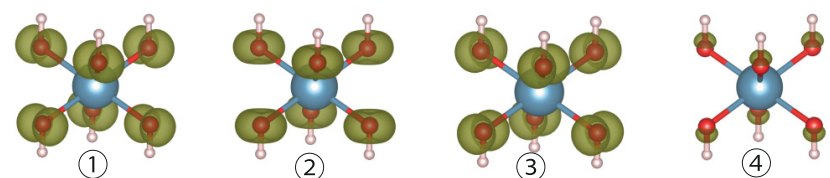

FIG. 5. (Color online) (a) Electronic band dispersion, (b) partial DOS, and (c) band and $k$-point decomposed charge densities of the bulk $\mathrm{Ca}(\mathrm{OH})_{2}$. The charge densities are the band edges indicated in panel (a), with isovalues kept constant. (d) Electronic band structure, (e) partial DOS (f) with (dashed line) and without (solid line) spin-orbit coupling band structure around the $\Gamma$ point, and $(\mathrm{g})$ band and $k$-point decomposed charge densities for monolayer $\mathrm{Ca}(\mathrm{OH})_{2}$. The charge densities at the band edges those indicated in panel (d); isovalues are kept constant and are the same as those in panel (c).

As shown in Fig. 5(a), the valence-band maximum (VBM) and the conduction-band minimum $(\mathrm{CBM})$ are located at the $\Gamma$ point. The partial DOS shown in Fig. 5(b) indicates that the major contribution to the states at the valence and conduction band edges originates from the $\mathrm{O}$ atoms, while deeper in the conduction band, states are mainly composed of the orbitals of $\mathrm{Ca}$. The orbital character of a state at a particular band can also be deduced from a band and $k$-point decomposed charge density. As seen from Fig. 5(c), edges in the top of VBM have $\mathrm{O} p_{x}$ and $\mathrm{O} p_{y}$ orbital character, and 


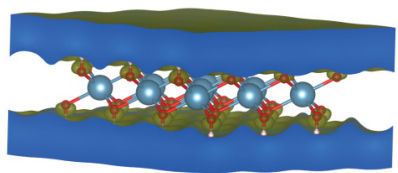

(a) Monolayer $E_{\mathrm{cl}}{ }^{\mathrm{\Gamma}}$

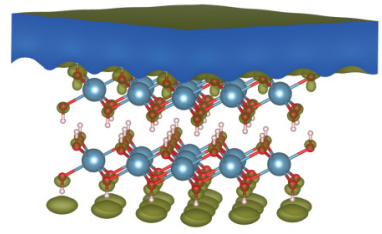

(c) Bilayer $E_{\mathrm{cl}}{ }^{\mathrm{\Gamma}}$

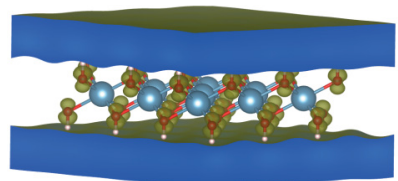

(b) Monolayer $E_{\mathrm{c} 2}{ }^{\Gamma}$

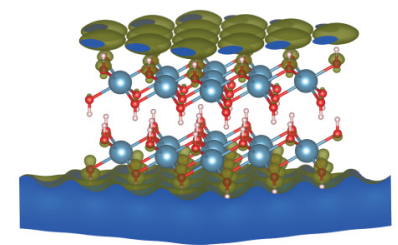

(d) Bilayer $E_{\mathrm{c} 2}{ }^{\mathrm{T}}$
FIG. 6. (Color online) Two lowest conduction band charge densities of monolayer and bilayer $\mathrm{Ca}(\mathrm{OH})_{2}$ at the $\Gamma$ point.

the hybridization of these states are also shown in the same figure. While the CBM has some $p_{z}$ orbital character from the $\mathrm{O}$ atoms, but as the energy of the state increases, the $d$ orbitals from $\mathrm{Ca}$ atom start to contribute, see (8) in the same figure.

Electronic properties of $\mathrm{Ca}(\mathrm{OH})_{2}$ are quite different from similar two-dimensional graphene-like structures. Unlike TMDCs (such as $\mathrm{MoS}_{2}$ and $\mathrm{WSe}_{2}$ ) that exhibit indirect-todirect band gap crossover when going from bulk to a monolayer structure, $\mathrm{Ca}(\mathrm{OH})_{2}$ is a direct band gap semiconductor which is independent of the number of layers. Although the energy band gap at the $\Gamma$ point decreases from 4.03 to $3.67 \mathrm{eV}$ for a monolayer structure, electronic dispersion of the valence band edge remains almost unchanged; see Fig. 5(d). As shown in Fig. 5(e) the conduction states mainly originate from $\mathrm{Ca}$ atoms, while the valence states are mainly composed of the orbitals of $\mathrm{O}$ atoms.

Our magnetic state analysis shows that, unless a defect is formed in or on the structure, there is no spin polarization in the ground state of both bulk and monolayer $\mathrm{Ca}(\mathrm{OH})_{2}$. Therefore, $\mathrm{Ca}(\mathrm{OH})_{2}$ is a nonmagnetic insulator regardless of its dimension for the structure.

Moreover, it was seen that the spin-orbit interaction has no considerable effect on the bond lengths and the overall electronic dispersion [except for a $26 \mathrm{meV}$ splitting in the VBM at the $\Gamma$ point, see Fig. 5(f)]. Due to the presence of inversion symmetry of $\mathrm{Ca}(\mathrm{OH})_{2}$, the degeneracy of spin-up and spin-down states still remains, this is also confirmed by the results of our calculation.

Band and $k$-point decomposed charge density in Fig. 5 are kept with the same isosurface level for comparison. However, as we further reduced the isosurface level at (4) in Figs. 5(d) and $5(\mathrm{~g})$, which is the lowest conduction band of the monolayer at the $\Gamma$ point: $E_{c 1}^{\Gamma}$, charge density forms a planar state parallel to the layer on both sides; see Fig. 6(a), this is also the case for the second-lowest non-spin-resolved conduction band at the same $k$ point: $E_{c 2}^{\Gamma}$; see Fig. 6(b). These two states are important due to their unique character and because their energy is right below the ionization energy. Such exceptional states having freeelectron-like dispersion were reported before [46] for doped graphite. To study the trend in these states, the same states were plotted for bilayer $\mathrm{Ca}(\mathrm{OH})_{2}$, see Figs. $6(\mathrm{c})$ and $6(\mathrm{~d}) . E_{c 1}^{\Gamma}$ and
$E_{c 2}^{\Gamma}$ have lower energies than the ionization energy. Therefore, electrons are still close and bond to both sides of monolayers as seen from charge density. In the case of bilayer, interestingly, these states appear only on one side of the bilayer.

\section{MECHANICAL PROPERTIES OF Ca(OH)}

Following the computational methodology described in Sec. II, we present quantities that describe the mechanical properties of $\mathrm{Ca}(\mathrm{OH})_{2}$ in Table II.

At first, the in-plane Young's modulus of the bulk structure is calculated. Bulk $\mathrm{Ca}(\mathrm{OH})_{2}$ has an in-plane Young's modulus $(55.0 \mathrm{~N} / \mathrm{m})$ and in-plane shear modulus $(21.23 \mathrm{~N} / \mathrm{m})$. Both these quantities indicate a flexible nature to in-plane tensile and shear deformation of bulk $\mathrm{Ca}(\mathrm{OH})_{2}$. In addition, bulk $\mathrm{Ca}(\mathrm{OH})_{2}$ has an in-plane Poisson ratio of 0.30. Additionally, the value of the in-plane stiffness for bulk $\mathrm{Ca}(\mathrm{OH})_{2}$ is calculated to be $60.1 \mathrm{~J} / \mathrm{m}^{2}$.

If we go from bulk to bilayer $\mathrm{Ca}(\mathrm{OH})_{2}$, we see a reduction in either the in-plane Young's modulus or the in-plane shear modulus, which are 50.7 and $19.16 \mathrm{~N} / \mathrm{m}$, respectively. The in-plane Poisson ratio, on the other hand, is slightly increased to 0.32 and becomes more spongy like as opposed to more cork-like in character [47]. In addition, the in-plane stiffness of bilayer $\mathrm{Ca}(\mathrm{OH})_{2}$ is calculated to be $55.6 \mathrm{~J} / \mathrm{m}^{2}$.

We found that monolayer $\mathrm{Ca}(\mathrm{OH})_{2}$ has a quite low inplane Young's modulus $(50.7 \mathrm{~N} / \mathrm{m})$ when compared to $\mathrm{BN}$ $(278.2 \mathrm{~N} / \mathrm{m})$. The in-plane Poisson ratio $(0.33)$ and the inplane shear modulus $(19.08 \mathrm{~N} / \mathrm{m})$ of the monolayer are similar to those of bilayer and $\mathrm{BN}$, which are 0.22 and $113.5 \mathrm{~N} / \mathrm{m}$, respectively. The calculated in-plane stiffness of monolayer $\mathrm{Ca}(\mathrm{OH})_{2}$ is $53.2 \mathrm{~J} / \mathrm{m}^{2}$.

\section{VIBRATIONAL CHARACTERISTICS OF MONOLAYER Ca(OH)}

Lastly, for the analysis of the vibrational spectrum and further examination of the dynamical stability of monolayer $\mathrm{Ca}(\mathrm{OH})_{2}$, we performed a calculation of the phonon spectrum using both the first-principles small displacement methodology (SDM) [48] and density functional perturbation methodology (DFPT) [49]. Here the nonquadratic dispersion of the flexural mode around the zone center is directly related to the insufficient fast Fourier transform (FFT) grid along the vacuum direction. It is seen from Fig. 7 that, similar to the Raman shift measurements observed from the bulk crystal structure, the monolayer material also has high-frequency $\mathrm{OH}$ stretching modes at $3700-3800 \mathrm{~cm}^{-1}$.

Further analysis of the analysis of phonon branches shows that the decomposition of the vibration representation of optical modes at the zone center is $\Gamma=4 E_{u}+2 A_{2 u}+4 E_{g}+$ $2 A_{1 g}$. As shown in the right panel of Fig. 7, there are four Raman-active phonon branches around 240, 350, 390, and $3700-3800 \mathrm{~cm}^{-1}$. It is also worth noting that as opposed to other TMDCs structures that have the 1T phase, the presence of $\mathrm{H}$ atoms results in the existence of two different $E_{g}$ and $A_{1 g}$ modes. Here the phonon dispersion have real eigenfrequencies in the whole Brillouin zone, which is another indication of the stability of monolayer $\mathrm{Ca}(\mathrm{OH})_{2}$. 


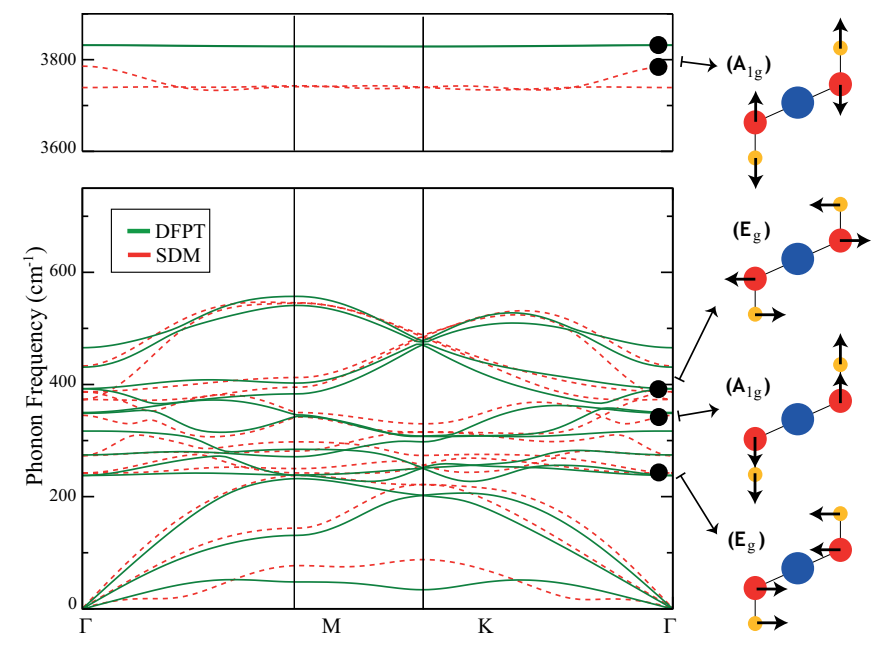

FIG. 7. (Color online) Phonon dispersion of monolayer $\mathrm{Ca}(\mathrm{OH})_{2}$.

Comparison of the vibrational spectrum with the experimentally observed Raman spectrum of bulk $\mathrm{Ca}(\mathrm{OH})_{2}$ reveals that two prominent Raman peaks at 282 and $357 \mathrm{~cm}^{-1}$ are identified as the $\mathrm{E}_{2 g}$ and $A_{1 g}$ vibrational modes, respectively. The high-frequency peak $A_{1 g}$ matches well the previously reported experimental and theoretical data, however, $\mathrm{E}_{2 g}$ shows slight differences in peak position. For example, Padanyi et al. [50] observed an $\mathrm{E}_{2 g}$ peak at around $260 \mathrm{~cm}^{-1}$ while reports from Schmid et al. [51] agrees well with our results. Accordingly, observed variations in the $\mathrm{E}_{2 g}$ peak might be linked to differences in the phase or to hidden phases introduced during the growth.

\section{DISCUSSION}

By performing first principles calculations on bulk, bilayer, and monolayer $\mathrm{Ca}(\mathrm{OH})_{2}$ and experimental confirmation of the bulk crystal layered structure, we predicted several important properties of this material and its stability. We found that (i) $\mathrm{Ca}(\mathrm{OH})_{2}$ crystals are environmentally stable and their stable structures can be synthesized by experimental methods. (ii) Experimentally, we also demonstrated that $\mathrm{Ca}(\mathrm{OH})_{2}$ crystals can be grown in layered form and also be exfoliated on arbitrary substrates. (iii) The dimensionality of $\mathrm{Ca}(\mathrm{OH})_{2}$ will not change the electronic, structural, and magnetic properties qualitatively, nevertheless intrinsic mechanical stiffness of each layer will become slightly stiffer as the system goes from monolayer to bilayer. (iv) The interlayer interaction is mainly a vdW dispersion-type force, and the strength of the interaction is stronger than that of similar layered materials (e.g., $\mathrm{MoS}_{2}$ and graphite). (v) Monolayer $\mathrm{Ca}(\mathrm{OH})_{2}$ remains dynamically stable even at high temperatures. (vi) The conduction states which have a free-like character may be utilized for high-mobility electron transfer.

We believe that the stable structure and the unique electronic properties of ultrathin $\mathrm{Ca}(\mathrm{OH})_{2}$ will trigger interest in this class of materials.

\section{ACKNOWLEDGMENTS}

This work was supported by the Flemish Science Foundation (FWO-Vl) and the Methusalem foundation of the Flemish government. Computational resources were provided by TUBITAK ULAKBIM, High Performance and Grid Computing Center (TR-Grid e-Infrastructure). H.S. is supported by a FWO Pegasus Long Marie Curie Fellowship.
[1] K. S. Novoselov, A. K. Geim, S. V. Morozov, D. Jiang, Y. Zhang, S. V. Dubonos, I. V. Grigorieva, and A. A. Firsov, Science 306, 666 (2004).

[2] K. S. Novoselov, D. Jiang, F. Schedin, T. J. Booth, V. V. Khotkevich, S. V. Morozov, and A. K. Geim, Proc. Natl. Acad. Sci. USA 102, 10451 (2005).

[3] A. K. Geim and K. S. Novoselov, Nat. Mater. 6, 183 (2007).

[4] G. G. Guzmán-Verri and L. C. Lew Yan Voon, Phys. Rev. B 76, 075131 (2007).

[5] S. Cahangirov, M. Topsakal, E. Akturk, H. Sahin, and S. Ciraci, Phys. Rev. Lett. 102, 236804 (2009).

[6] M. Houssa, G. Pourtois, V. V. Afanas'ev, and A. Stesmans, Appl. Phys. Lett. 96, 082111 (2010).

[7] H. Sahin, S. Cahangirov, M. Topsakal, E. Bekaroglu, E. Akturk, R. T. Senger, and S. Ciraci, Phys. Rev. B 80, 155453 (2009).

[8] A. H. C. Neto and K. Novoselov, Rep. Prog. Phys. 74, 082501 (2011).

[9] C. Ataca, H. Sahin, and S. Ciraci, J. Phys. Chem. C 116, 8983 (2012).

[10] R. M. D. Lieth and J. C. Terhell, in Preparation and Crystal Growth of Materials with Layered Structures, edited by R. M. D. Lieth (Reidel, Dordrecht, 1976).

[11] C. Meade and R. Jeanloz, Geophys. Res. Lett. 17, 1157 (1990).
[12] M. B. Kruger, Q. Williams, and R. Jeanloz, J. Chem. Phys. 91, 5910 (1989).

[13] J. H. Nguyen, M. B. Kruger, and R. Jeanloz, Phys. Rev. Lett. 78, 1936 (1997).

[14] S. Raugei, P. L. Silvestrelli, and M. Parrinello, Phys. Rev. Lett. 83, 2222 (1999).

[15] T. S. Duffy, C. Meade, Y. Fei, H. K. Mao, and R. J. Hemley, Am. Mineral. 80, 222 (1995).

[16] S. Ekbundit, K. Leinenweber, J. L. Yarger, J. S. Robinson, M. Verhelst-Voorhees, and G. H. Wolf, J. Solid State Chem. 126, 300 (1996).

[17] K. Catalli, S.-H. Shim, and V. B. Prakapenka, Geophys. Res. Lett. 35, L05312 (2008).

[18] R. Iizuka, H. Kagi, K. Komatsu, D. Ushijima, S. Nakano, A. Sano-Furukawa, T. Nagai, and T. Yagi, Phys. Chem. Miner. 38, 777 (2011).

[19] B. Winkler, V. Milman, B. Hennion, M. C. Payne, M.-H. Lee, and J. S. Lin, Phys. Chem. Miner. 22, 461 (1995).

[20] Ph. Baranek, A. Lichanot, R. Orlando, and R. Dovesi, Chem. Phys. Lett. 340, 362 (2001).

[21] K. Azuma, T. Oda, and S. Tanaka, Comput. Theor. Chem. 963, 215 (2011).

[22] Ph. D'Arco, M. Causà, C. Roetti, and B. Silvi, Phys. Rev. B 47, 3522 (1993). 
[23] A. Pishtshev, S. Zh. Karazhanov, and M. Klopov, Comput. Mater. Sci. 95, 693 (2014).

[24] A. Pishtshev, S. Zh. Karazhanov, and M. Klopov, Solid State Commun. 193, 11 (2014).

[25] W. Ren and H.-M. Cheng, Nat. Nanotechnol. 9, 726 (2014).

[26] Y. Yu, C. Li, Y. Liu, L. Su, Y. Zhang, and L. Cao, Sci. Rep. 3, 1866 (2013).

[27] G. Kresse and J. Hafner, Phys. Rev. B 47, 558(R) (1993).

[28] G. Kresse and J. Hafner, Phys. Rev. B 49, 14251 (1994).

[29] G. Kresse and J. Furthmüller, Comput. Mater. Sci. 6, 15 (1996).

[30] G. Kresse and J. Furthmüller, Phys. Rev. B 54, 11169 (1996).

[31] J. P. Perdew, K. Burke, and M. Ernzerhof, Phys. Rev. Lett. 77, 3865 (1996).

[32] J. P. Perdew, K. Burke, and M. Ernzerhof, Phys. Rev. Lett. 78, 1396 (1997).

[33] P. E. Blöchl, Phys. Rev. B 50, 17953 (1994).

[34] G. Kresse and D. Joubert, Phys. Rev. B 59, 1758 (1999).

[35] S. Grimme, J. Comput. Chem. 27, 1787 (2006).

[36] W. Tang, E. Sanville, and G. Henkelman, J. Phys.: Condens. Matter 21, 084204 (2009).

[37] E. Sanville, S. D. Kenny, R. Smith, and G. Henkelman, J. Comput. Chem. 28, 899 (2007).

[38] G. Henkelman, A. Arnaldsson, and H. Jónsson, Comput. Mater. Sci. 36, 354 (2006).
[39] See, e.g., J. F. Nye, Physical Properties of Crystals (Oxford University Press, Oxford, 1985), p. 141.

[40] M. Levy, H. E. Bass, and R. R. Stern, Handbook of Elastic Properties of Solids Liquids and Gases (Academic, San Diego, 2000), Vol. 1.

[41] L. Desgranges, D. Grebille, G. Calvarin, G. Chevrier, N. Floquet, and J. C. Niepce, Acta Crystallogr., Sect. B: Struct. Sci. 49, 812 (1993).

[42] W. R. Busing and H. A. Levy, J. Chem. Phys. 26, 563 (1957).

[43] M. Topsakal, E. Akturk, and S. Ciraci, Phys. Rev. B 79, 115442 (2009).

[44] Q. Peng, W. Ji, and S. De, Comput. Mater. Sci. 56, 11 (2012).

[45] C. Ataca, M. Topsakal, E. Akturk, and S. Ciraci, J. Phys. Chem. C 115, 16354 (2011).

[46] M. Posternak, A. Baldereschi, A. J. Freeman, and E. Wimmer, Phys. Rev. Lett. 52, 863 (1984); M. Posternak, A. Baldereschi, A. J. Freeman, E. Wimmer, and M. Weinert, ibid. 50, 761 (1983).

[47] G. N. Greaves, A. L. Greer, R. S. Lakes, and T. Rouxel, Nat. Mater. 10, 823 (2011).

[48] D. Alfe, Comput. Phys. Commun. 180, 2622 (2009).

[49] S. Baroni, S. de Gironcoli, A. Dal Corso, and P. Giannozzi, Rev. Mod. Phys. 73, 515 (2001).

[50] Z. V. Padanyi, Solid State Commun. 8, 541 (1970).

[51] T. Schmid and P. Dariz, J. Raman Spectrosc. 46, 141 (2015). 